\title{
Norms and equivalences for MoCA-30, MoCA-22, and MMSE in the oldest-old
}

\author{
Zarui A. Melikyan ${ }^{1}$ (D) Michael Malek-Ahmadi ${ }^{2}$ (D) Kathleen O'Connor $^{2} \cdot$ Alireza Atri $^{2,3,4} \cdot$ Claudia H. Kawas $^{1,5,6}$. \\ María M. Corrada ${ }^{1,5,7}$
}

Received: 23 October 2020 / Accepted: 10 May 2021 / Published online: 29 May 2021

(c) The Author(s) 2021

\begin{abstract}
Background Cognitive screening is important for the oldest-old (age 90+). This age group is the fastest growing and has the highest risk of dementia. However, norms and score equivalence for screening tests are lacking for this group.

Aims To provide norms and score equivalence for commonly used cognitive screening tests for the oldest-old.

Methods Data on 157 participants of the Center for Healthy Aging Longevity Study aged 90+ were analyzed. First, we derived norms for (1) subtests and cognitive domains of the in-person Montreal Cognitive Assessment having a maximum score of 30 (MoCA-30) and (2) the total MoCA-22 score, obtained from the in-person MoCA-30 by summing the subtests that do not require visual input to a maximum score of 22. These norms were derived from 124 participants with a MiniMental State Examination (MMSE) $\geq 27$. Second, we derived score equivalences for MMSE to MoCA-30 and MoCA-22, and MoCA-30 to MoCA-22 using equipercentile equating method with log-linear smoothing, based on all 157 participants. Results MoCA-22 total score norms are: mean =18.3(standard deviation=2.2). An MMSE score of 27 is equivalent to a MoCA-30 score of 22 and a MoCA-22 score of 16.

Discussion and conclusions Subtest, domain and MoCA-22 norms will aid in evaluation of the oldest-old who cannot complete the MoCA-30 or are tested over the phone. The equivalences of the three cognitive tests (MMSE, MoCA-30, MoCA22) in the oldest-old will facilitate continuity of cognitive tracking of individuals tested with different tests over time and comparison of the studies that use different cognitive tests.
\end{abstract}

Keywords Oldest-old $\cdot 90+\cdot$ Score conversion $\cdot$ MMSE $\cdot$ MoCA-30 $\cdot$ MoCA-22

Zarui A. Melikyan

zmelikya@uci.edu

1 Institute for Memory Impairments and Neurological Disorders, University of California Irvine, 1511 Hewitt Hall, 843 Health Sciences Road, Irvine, CA 92697, USA

2 Banner Sun Health Research Institute, Sun City, AZ, USA

3 Center for Brain/Mind Medicine, Department of Neurology, Brigham and Women's Hospital, Boston, MA, USA

4 Harvard Medical School, Boston, MA, USA

5 Department of Neurology, University of California Irvine, Irvine, CA, USA

6 Department of Neurobiology and Behavior, University of California Irvine, Irvine, CA, USA

7 Department of Epidemiology, University of California Irvine, Irvine, CA, USA

\section{Introduction}

Cognitive screening of the oldest-old (age 90+) has become increasingly important, because this age group has the highest risk of dementia [1] and its projected growth in the coming decades is rapid [2]. However, cognitive testing of this age group is challenging. First, sensory and cognitive impairments make many of the oldest-old unable to complete all subtests of in-person screening measures, which makes calculation of the total score and its comparison to normative values impossible. In such situations, subtest and domain norms allow for evaluation of completed subtests. In that vein, subtest and domain norms in younger-old (older adults younger than 90 years) have been published for one of the most frequently used screening measures, the in-person Montreal Cognitive Assessment that has a maximum possible score of 30 (MoCA-30) [3-6]. Additionally, MoCA-Blind [7], that includes MoCA-30 subtests that do 
not require visual input and has a maximum possible score of 22, was developed to enable in-person cognitive screening of individuals with visual impairment. MoCA-Blind normative cut-points, to distinguish cognitively normal from cognitively impaired younger-old, were published for the sum of in-person MoCA-30 subtests included in MoCA-Blind [8]. Henceforth, the sum of in-person MoCA-30 subtests included in MoCA-Blind, that has maximum possible score of 22, is called MoCA-22. The second challenge in testing the oldest-old is that prevalent frailty and other comorbidities prevent many of them from travelling to testing sites, which makes telephone screening a method of choice. To address the need for telephone cognitive screening, Telephone MoCA [7], identical to MoCA-Blind with slightly modified testing procedures to accommodate telephone testing, was developed and normative cut-points published for younger-old [9-12]. However, no norms for MoCA-30 subtests and domains or MoCA-Blind/ Telephone MoCA total score have been published for the oldest-old.

Recently, many clinical and research settings have switched to using the MoCA-30/ MoCA-Blind/ Telephone MoCA from the Mini-Mental State Exam (MMSE) [13], another common screening measure. The MoCA-30 is more sensitive to mild forms of cognitive impairment, has a higher diagnostic accuracy than the MMSE [14-17], and is available at no cost. To facilitate continuity of cognitive tracking in individuals tested with MMSE, MoCA-30, and MoCA-Blind/ Telephone MoCA at different times and to provide comparability of data among multiple studies and trials, the ability to equate scores of these three tests is necessary. Equating MMSE to MoCA-30 in the younger-old shows that, although these two tests have identical score range (0-30), they do not have one-to-one correspondence. Rather, higher MMSE scores correspond to lower MoCA-30 scores, likely because some of MoCA-30 subtests are more challenging $[16,18,19]$. While two studies have equated MMSE and MoCA-30 to Telephone MoCA and MoCA-22 in the younger-old $[19,20]$, equivalencies for the oldestold have not been reported. Lacking Telephone MoCA data, and given that equivalence of Telephone MoCA and MoCA22 was recently demonstrated [20], we used the MoCA-22 as the next best option to equate it with the MMSE and MoCA-30.

The aims of this study are to provide in the oldest-old: (1) norms for subtests and cognitive domains of in-person MoCA-30 and norms for MoCA-22 total score derived from in-person MoCA-30 by summing its subtests that do not require visual input, and (2) score equivalence of MMSE to MoCA-30 and MoCA-22, and MoCA-30 to MoCA-22.

\section{Methods}

\section{Study procedures}

We report on a subset of participants of the ongoing Center for Healthy Aging Longevity Study [21]. Since its inception in 2007, the study has collected demographic, physical, and cognitive data on older adults aged $50-110$ years to explore factors associated with increased longevity and healthy aging. Study enrollment occurs continuously and participants are recruited through advertisements, community talks, referrals from current study participants throughout the state of Arizona, predominantly in Sun Cities of the northwest Phoenix metropolitan area. All participants are community-dwelling, independent individuals deemed to be cognitively unimpaired based on structured interviews, testing, and study protocol assessment, and supported by self-reported medical history of no dementia, and no cognitive, neurological or other diagnoses with high likelihood to cause cognitive impairment. All individuals reported independence in personal, home and community affairs and no difficulties or support for activities of daily living that related to cognition. Participants are assessed annually either at the Banner Sun Health Research Institute or at their residences. Cognitive diagnosis is not assigned as part of the research visit.

The study was approved by the Western Institutional Review Board and all participants provided signed informed consent. Research was completed in accordance with the Helsinki Declaration.

\section{Participants and data items}

We requested from the Center for Healthy Aging Longevity Study data on participants who were aged 90 or older and who had completed both MoCA-30 and MMSE inperson at their first cognitive testing. We requested MoCA30 (version 7.1) [7] total and subtest scores, MMSE total scores, and total score for the Center for Epidemiologic Studies Depression Scale (CES-D) [24]. There were no missing scores in the data provided. In addition, we requested information on sex, race, age at first visit, residence type (own housing, independent living facility with congregate meals, assisted living facility, nursing home or care center), and education. Health information was not available. Data on 157 eligible participants were provided on Feb 27, 2020.

Normative data were derived for a subgroup of 124 participants with MMSE $\geq 27$, a cut point that differentiates normal cognition from mild cognitive impairment and dementia [22, 23]. Data from the 157 participants were 
used to provide score equivalence of MMSE to MoCA-30 and MoCA-22, and MoCA-30 to MoCA-22.

To obtain the total MoCA-22 score, we summed the scores for MoCA-30 subtests that that do not require visual input (i.e., digit span forward, digit span backwards, letter A tapping, serial 7s, sentence repetition, letter F fluency, similarities, delayed recall, orientation to time and place), as was done in previous publications $[19,20]$ (Table 1). One point was added to total MoCA-30 and MoCA-22 scores for individuals with $\leq 12$ years of education according to the manual.

\section{Data analysis}

To provide norms for MoCA-30 domains and subtests and for MoCA-22 total score, we used a subgroup of participants with MMSE $\geq 27$ (the normative subgroup). Norms are reported as means, standard deviations, and percent of participants who obtained the highest possible domain or subtest score. For example, the maximum score for visuospatial/executive domain is five and we provide the percent of participants from the normative subgroup who obtained a score of five on this domain. Subtest norms are reported as the percent of participants that obtained highest possible score on each subtest. For example, maximum score on the Trail Making subtest is one and we provide the percent of participants from the normative subgroup who obtained a score of one on this subtest. Norms are provided for the following age categories: 90-91, 92-94, and $\geq 95$ years, which were chosen because they contain approximately equal number of participants and correspond to our previous normative publications [25, 26]. The effect of age group on the scores was assessed by Kruskal-Wallis test.

To derive equivalence of MMSE to MoCA-30 and MoCA-22, and MoCA-30 to MoCA-22 total scores, we used the equipercentile equating method with log-linear smoothing [16-19, 27-30]. This method equates scores from two measures based on the equivalency of their corresponding percentile ranks [31]. The log-linear smoothing of the raw scores before the equipercentile equating is used, because raw score distributions are often irregular, i.e., the percentage of the test takers with a given score does not change gradually as the score increases or decreases. These irregularities produce irregularities in equipercentile equating adjustment and those irregularities, in turn, do not generalize to other groups of test takers. The log-linear smoothing technique mitigates this issue by replacing the observed score distribution with a distribution that has the same location, spread and shape, but not the irregularities.

Demographic data are expressed as means, standard deviations, and ranges for numeric variables, and percentage and frequencies for the categorical variables.

All analyses were done using R-Studio version 1.1.414 with $\mathrm{R}$ version 3.4.3. [32]. Log-linear smoothing and equipercentile equating were done using the 'equate' R library [33].
Table 1 Cognitive domains and subtests included in MoCA-30 and MoCA-22

\begin{tabular}{llll}
\hline Cognitive domain & Subtest & MoCA-30 & MoCA-22 \\
\hline Visuospatial/Executive & Trail Making Test & + & \\
& Copy cube & + & \\
& Draw clock & + & \\
Naming & Picture naming & + & + \\
Attention & Digit Span forward & + & + \\
& Digit Span backwards & + & + \\
& Letter A tapping & + & + \\
& Serial 7s & + & + \\
Language & Sentence repetition & + & + \\
Abstraction & Letter F fluency & + & + \\
Memory & Similarities & + & + \\
Orientation & Delayed recall & + & + \\
\hline
\end{tabular}

MoCA-30 Montreal Cognitive Assessment administered in-person with a maximum possible score of 30, MoCA-22 Version of MoCA-30 that excludes items that require visual input and has a maximum possible score of 22

"+" means the subtest is included in the MoCA-30 or MoCA-22 


\section{Results}

\section{Characteristics of the study groups: demographics and total cognitive test scores}

The entire group of 157 participants had a mean age of 93.5 years (range: 90-102). Most participants were women $(67 \%)$, well-educated (mean years $=15.1)$, and lived independently (94\%). All participants were White (Table 2).

The demographic characteristics of the normative subgroup of 124 participants with MMSE $\geq 27$ were comparable to those of the entire group. In the normative subgroup, the mean age was 93.5 years (range: 90-101); most were women $(73 \%)$, well-educated (mean years $=15.3$ ), and lived independently (96\%). All the normative group participants were White (Table 2).

In the entire group of 157 participants the mean MoCA30 score was 24.2, the mean MoCA-22 score was 17.7, and the mean MMSE score was 28.1. In the normative subgroup, the mean MoCA-30 score was 24.8, the mean MoCA-22 score 18.3, and the mean MMSE score was 28.8, each about 0.7 higher than in the entire group (Table 2). Mean total MoCA-30, MoCA-22, and MMSE scores did not significantly differ by residence type in the entire group or in the normative subgroup (Table 3 ).

\section{Norms for MoCA-30 subtest and domain scores and MoCA-22 total score}

The percent of participants with the highest possible domain scores was lowest for memory (15\% of participants) and visuospatial/executive ( $25 \%$ of participants). Conversely, the percent was highest for the domains of orientation ( $89 \%$ of participants) and naming (78\% of participants) (Table 4$)$. The MoCA-22 normative group mean total score is 18.3 and the standard deviation is 2.2. The Supplement contains norms for each domain by age category and overall (Supplementary Table 1), and percent of participants with highest subtest scores by age category and overall (Supplementary Table 2).

\section{Equivalences of MMSE, MoCA-30, and MoCA-22 Scores}

The equivalences of MMSE to MoCA-30 and MoCA-22, and MoCA-30 to MoCA-22 scores using the equipercentile equating method with log-linear smoothing are shown in Fig. 1. An MMSE score of 27 was equivalent to a MoCA30 score of 22 and to a MoCA-22 score of 16. A MoCA-30 score of 22 is equivalent to a MoCA-22 score of 16.

Table 2 Characteristics of study participants for the entire group and the normative subgroup defined as MMSE $\geq 27$

\begin{tabular}{lcc}
\hline Characteristic & $\begin{array}{l}\text { Entire group } \\
(N=157) \\
N(\%)\end{array}$ & $\begin{array}{l}\text { Normative subgroup } \\
N=124)\end{array}$ \\
\hline Sex, women & $105(66.9)$ & $90(72.6)$ \\
Age group, years & $45(28.7)$ & $41(33.1)$ \\
$90-91$ & $57(36.3)$ & $43(34.7)$ \\
$92-94$ & $55(35.0)$ & $40(32.3)$ \\
$\geq 95$ & & $124(100)$ \\
Race, White & $157(100)$ & $95(77.2)$ \\
Education level, > high school & $120(76.9)$ & $77(62.1)$ \\
Residence type & $92(58.6)$ & $41(33.1)$ \\
Own residence & $55(35.0)$ & $5(4.0)$ \\
Independent living with congregate meals & $9(5.7)$ & \\
Assisted living, nursing home or care center & & $93.5(2.9)[90-101]$ \\
& Mean (SD) [Range] & $15.3(1.7)[9-18]$ \\
\hline Age, years & $93.5(2.9)[90-102]$ & $24.8(2.5)[19-30]$ \\
Education, years & $15.1(1.8)[9-18]$ & $18.3(2.2)[13-22]$ \\
MoCA-30 & $24.2(2.8)[17-30]$ & $28.8(1.0)[27-30]$ \\
MoCA-22 & $17.7(2.4)[11-22]$ & $4.7(4.7)[0-27]$ \\
MMSE & $28.1(1.8)[21-30]$ & \\
CES-D & $4.9(4.8)[0-27]$ & \\
\hline
\end{tabular}

MoCA-30 Montreal Cognitive Assessment administered in-person with a maximum possible score of 30

MoCA-22 Version of MoCA-30 that excludes items that require visual input and has a maximum possible score of 22

MMSE Mini-Mental State Examination, CES-D Center for Epidemiologic Studies Depression Scale 
Table 3 Mean (standard deviation) of cognitive test scores by place of residence
Table 4 Mean, standard deviation (SD), and range of total cognitive test and domain scores for MoCA30 and MoCA-22 for the normative subgroup defined as MMSE $\geq 27$

\begin{tabular}{llllll}
\hline & & Own residence & $\begin{array}{l}\text { Independent living } \\
\text { with congregate } \\
\text { meals }\end{array}$ & $\begin{array}{l}\text { Assisted living, } \\
\text { nursing home or care } \\
\text { center }\end{array}$ & $p$ value \\
\hline Entire group & MoCA-30 & $24.4(2.6)$ & $24.0(2.8)$ & $22.1(3.3)$ & 0.18 \\
& MoCA-22 & $18.0(2.3)$ & $17.4(2.5)$ & $15.7(2.9)$ & 0.06 \\
Normative subgroup & MoCA-30 & $24.8(2.5)$ & $24.9(2.5)$ & $26.9(2.0)$ & 0.19 \\
& MoCA-22 & $18.3(2.2)$ & $18.2(2.3)$ & $17.0(2.9)$ & 0.47 \\
& MMSE & $28.8(1.1)$ & $29.0(0.9)$ & $28.4(0.9)$ & 0.48 \\
\hline
\end{tabular}

MoCA-30 Montreal Cognitive Assessment administered in-person with a maximum possible score of 30;

MoCA-22 Version of MoCA-30 that excludes items that require visual input and has a maximum possible score of 22;

MMSE Mini-Mental State Examination;

$p$ value for difference among residential groups from Kruskal-Wallis test

\begin{tabular}{lrrll}
\hline $\begin{array}{l}\text { Test/domain } \\
\text { (possible score range) }\end{array}$ & Mean & SD & $\begin{array}{l}\text { Actual score } \\
\text { range }\end{array}$ & $\begin{array}{l}\text { Percent of participants with the } \\
\text { highest possible total or domain } \\
\text { score }\end{array}$ \\
\hline MoCA-30 (0-30) & 24.8 & 2.5 & $19-30$ & 2 \\
Visuospatial/executive (0-5) & 3.8 & 1.1 & $0-5$ & 25 \\
Naming (0-3) & 2.8 & 0.4 & $2-3$ & 78 \\
Attention (0-6) & 5.5 & 0.8 & $2-6$ & 67 \\
Language (0-3) & 2.4 & 0.7 & $1-3$ & 53 \\
Abstraction (0-2) & 1.7 & 0.6 & $0-2$ & 77 \\
Memory (0-5) & 2.6 & 1.7 & $0-5$ & 15 \\
Orientation (0-6) & 5.8 & 0.6 & $0-6$ & 89 \\
MoCA-22 (0-22) & 18.3 & 2.2 & $13-22$ & 6 \\
\hline
\end{tabular}

MoCA-30 Montreal Cognitive Assessment administered in-person with a maximum possible score of 30;

MoCA-22 Version of MoCA-30 that excludes items that require visual input and has a maximum possible score of 22

\section{Discussion}

This work provides norms for MoCA-30 subtests and domains, and MoCA-22 total score derived from oldestold adults with normal cognition defined as MMSE $\geq 27$. These norms allow interpretation of MoCA-30 performance for oldest-old individuals who cannot complete the entire test or were tested over the telephone. This study also provides in the oldest-old score equivalences of the three tests (MMSE, MoCA-30, MoCA-22) to facilitate comparison and conversion of scores between multiple centers and trials and within studies that used different tests at different times. The norms and score equivalences of these three tests provided in this paper are important in facilitating the use of screening measures in the oldest-old.

While both MMSE and MoCA-30 were administered in-person to participants in this study, MoCA-22 was not administered as a separate instrument. It was derived from the scores of the in-person MoCA-30 by summing those subtests that do not require visual input. Previous studies have demonstrated equivalence between the Telephone MoCA and the MoCA-22 [20], and similar reliability (area under the ROC curve) of these two measures to detect mild cognitive impairment [10]. Our results are particularly useful for evaluations that use Telephone MoCA or MoCA-Blind, given the absence of any information on these tests in the oldest-old.

Our total and domain norms for MoCA-30 and MoCA-22 total score generally correspond well to the two studies that published similar norms, albeit in younger-old $[4,5]$ (see Supplementary table 3 summarizing our norms and norms from these two studies). However, total MoCA-30 score and scores on visual-spatial/executive and memory domains are lower in our study than in the 1 study of 758 members of Swedish population-based cohort age $65-85$ years [4]. This difference is likely due to younger age and perhaps to the more rigorous procedure to determine cognitive status in the Swedish study. A third study that provides percent of cognitively normal older adults with the highest score on 

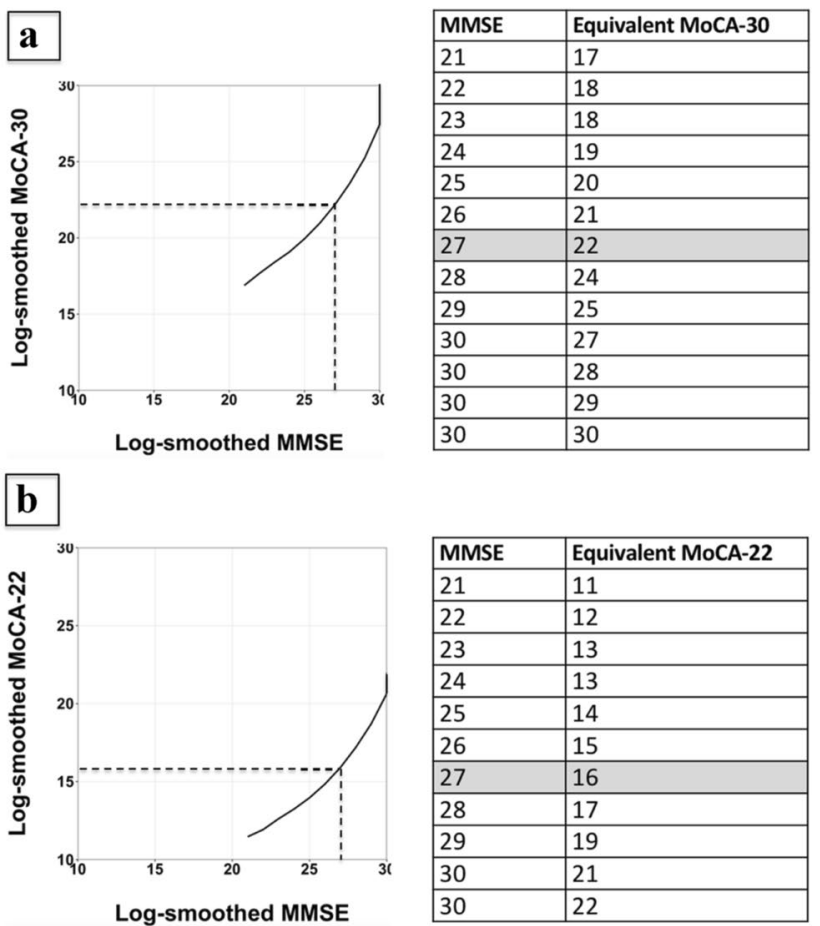

\begin{tabular}{|l|l|}
\hline MMSE & Equivalent MoCA-22 \\
\hline 21 & 11 \\
\hline 22 & 12 \\
\hline 23 & 13 \\
\hline 24 & 13 \\
\hline 25 & 14 \\
\hline 26 & 15 \\
\hline 27 & 16 \\
\hline 28 & 17 \\
\hline 29 & 19 \\
\hline 30 & 21 \\
\hline 30 & 22 \\
\hline
\end{tabular}

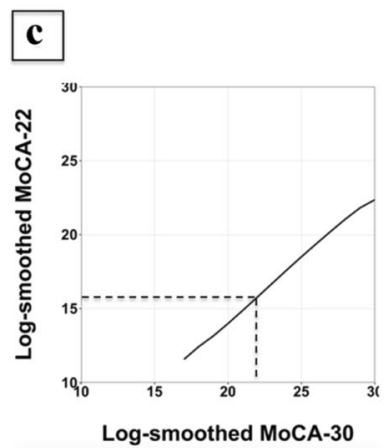

\begin{tabular}{|l|l|}
\hline MoCA-30 & Equivalent MoCA-22 \\
\hline 17 & 12 \\
\hline 18 & 12 \\
\hline 19 & 13 \\
\hline 20 & 14 \\
\hline 21 & 15 \\
\hline 22 & 16 \\
\hline 23 & 17 \\
\hline 24 & 18 \\
\hline 25 & 19 \\
\hline 26 & 19 \\
\hline 27 & 20 \\
\hline 28 & 21 \\
\hline 29 & 22 \\
\hline 30 & 22 \\
\hline
\end{tabular}

Fig. 1 a Mini-Mental State Examination (MMSE) mapped to Montreal Cognitive Assessment with a maximum possible score of 30 (MoCA-30) using equipercentile equating method. Dotted lines indicate that 27 points on the MMSE is equivalent to 22 points on the MoCA-30. These scores are also shaded on the table. b MMSE mapped to Montreal Cognitive Assessment with a maximum possible score of 22 (MoCA-22) using equipercentile equating method. Dotted lines indicate that 27 points on the MMSE is equivalent to 16 points on the MoCA-22. These scores are also shaded on the table. $\mathbf{c}$ MoCA30 mapped to MoCA-22 using equipercentile equating method. Dotted lines indicate that 22 points on MoCA- 30 is equivalent to 16 points on the MoCA-22. These scores are also shaded on the table

each MoCA-30 subtest also reports results that are similar to ours [3]. In our study, domain and subtest norms do not differ significantly by age group (Supplementary Tables 1 , 2). Given that age was used as categorical variable and that subtests often had narrow score ranges (e.g., some subtests had only two possible scores: 0 or 1), the study does not have the precision to observe the a priori expected age-related decrements in performance on all the tests. Until there is additional data to further delineate performance in the oldest-old, we suggest using total, rather than the age-groupspecific, subtest scores as a normative reference.

We determined the equivalence of MMSE to MoCA-30 and MoCA-22, and MoCA-30 to MoCA-22 scores using the equipercentile equating method as was done in most studies [16-20, 27-30]. We found that an MMSE score of 27 corresponds to a MoCA-30 score of 22, which is similar to other reports where MMSE score of 27 is equivalent to MoCA-30 scores of 21-24 [16-19, 27-30, 34-37]. These studies (reviewed in Supplementary Table 4) included younger-old participants with cognition ranging from normal to dementia.

The MMSE and MoCA-30 are both global cognitive screening measures with the same range of scores $(0-30)$, so it might be erroneously assumed that their scores have a oneto-one correspondence. However, the two tests emphasize different aspects of cognition. While the MMSE allocates more points on orientation (10 out of 30) than the MoCA30 (6 out of 30), the MoCA-30 places a greater emphasis on visuospatial domain (5 out of 30) than the MMSE (1 out of 30). As a consequence, it is not surprising that these two tests do not have a strictly linear relationship as demonstrated here and supported by previous evidence [18]. Also, some subtests in the MoCA-30 may be more challenging than those in the MMSE (e.g., delayed recall on the MoCA30 involves five words with a longer delay compared to the three words and shorter delay in MMSE), which contributes to lower MoCA-30 scores as reported here and in previous publications [16-18, 27, 36].

In this study, an MMSE score of 27 equates to a MoCA22 score of 16 . The only relevant publication we found equates MMSE score of 27 to MoCA-22 score of 17 (derived from MoCA-30) in a younger (mean age $=84$ years) group of 119 patients from general and geriatric medicine [19]. To put our finding in additional context, a MoCA-22 score of 16 , which could be suggested as a cut point for cognitive impairment because it corresponds to an MMSE cut point for cognitive impairment, is lower but generally comparable to the MoCA-Blind cut point of 18 or 19 suggested by the test author and validated in younger ages (55-85 years) [38], and to the Telephone MoCA cut-points of 17-19 suggested for younger-old (age range: 62-75 years) [8-11] (also reviewed in Supplementary table 5). We demonstrated that a MoCA-30 score of 22 equates to a MoCA-22 score of 16 , which is the same as that in a study of younger-old that equated the in-person MoCA-30 to the Telephone MoCA [20]. Similarly, in another study of younger-old, a MoCA-30 score of 22 equates to a MoCA-22 score of 17 derived from the in-person MoCA-30 [19].

This paper has several notable strengths. First, we report data on a relatively large sample of individuals aged $90+$. 
This allowed us to investigate the age effect on the test scores in the oldest-old. Second, we use equipercentile equating method that was previously used with younger-old and our results are comparable with those studies. Third, we report on widely used cognitive screening tests, MoCA-30 and MMSE. Fourth, normative data on screening subtests and domains for the oldest-old is reported for the first time.

We acknowledge several limitations. First, our sample represents mostly the upper part of the cognitive spectrum in the oldest-old; thus, we were not able to provide score equivalences for the lower functioning individuals. However, the high cognitive status of our participants makes our study comparable to others, which also have high prevalence of the upper spectrum of cognitive scores [28], and the agreement between our and other studies [3-5, 16-20, 30, 34] indicates high reliability of our findings. Second, generalizability of our findings to different racial and educational groups is limited as our sample is all White (compared to $88 \%$ in oldest-old in the U.S.) and highly educated (77\% in our study vs. $28 \%$ in the oldest-old in the U.S. have more than a high school education). At the same time, our sample of about two-thirds women, closely matches the sex distribution of the oldest-old in the U.S. (67\% in our group vs. $70 \%$ in oldest-old in the U.S.) [39]. Although norms derived from diverse populations are needed, in the absence of such norms for the oldest-old, these norms may be used with caution keeping in mind differential effect of health, educational and other factors that affect test performance in diverse populations [40]. Third, both MMSE and MoCA-30 were administered on the same day, with MMSE being administered before MoCA-30. The order of administration may have altered MoCA-30 performance either through learning effects for similar items or mental fatigue. Fourth, although studies show that test performance is related to health status, data on the health characteristics of our participants were not available to us. Fifth, although sample sizes for age subgroup norms are modest (about 40 per group), they are close to a desirable size of 50 that provides a stable estimate of population mean [41].

\section{Conclusions}

MoCA-30 domain and subtest norms and MoCA-22 norms for the oldest-old provided in this paper will facilitate evaluation of oldest-old individuals who are unable to complete the entire MoCA-30 or who are tested over the telephone. Score equivalences of MMSE, MoCA-30 and MoCA-22 in the oldest-old provided in this paper will help maintain continuity of care when multiple evaluations use different tests at different times and will allow data comparability among different studies.
Supplementary Information The online version contains supplementary material available at https://doi.org/10.1007/s40520-021-01886-z.

Acknowledgements The authors thank Prof. Vladimir Baranovsky, Ph.D. for valuable discussions on data analyses. The authors thank the participants and the staff of the Center for Healthy Aging Longevity Study at the Banner Sun Health Research Institute.

Author's contributions Conception/design: ZAM, MMC, and CHK. Data collection: MMA, KO, and AA. Data analysis and first draft: ZAM. Critical revisions for important intellectual content: all authors.

Funding This work was supported by the National Institute on Aging (C.H.K., M.M.C., Z.A.M. grant \# R01AG021055), the Arizona Department of Health Services, and Arizona Alzheimer's Consortium (M.M.A., K.O., A.A. grant \# CTR040636), and generous endowment and research and infrastructure support from The Sun Health Foundation and Banner Health (M.M.A., K.O., A.A.). The funding organizations had no role in the design or conduct of the study; collection, management, analysis, or interpretation of the data; in the writing of the manuscript; or in the decision to submit the manuscript for publication.

Data availability The dataset used for the current study is available from the corresponding author on reasonable request.

Code availability The $\mathrm{R}$ code used for the current study is available from the corresponding author on reasonable request.

\section{Declarations}

Conflict of interest C.H.K., M.M.C., Z.A.M., M.M.A., K.O. have no conflict of interest to declare. A.A. has received unrelated institutional grant funding from Alzheimer's Clinical Trials Consortium (ACTC), Alzheimer's Therapeutics Research Institute (ATRI), Alzheimer's Prevention Initiative (API), Arizona Alzheimer's Research Consortium, Global Alzheimer's Platform (GAP), Novartis, University of Indiana (with ATRI, NIH, Alzheimer's Association), University of Southern California (with ATRI, NIH, Alzheimer's Association or Department of Defense). A.A. has received consulting fees and honoraria from Acadia, Alzheimer's Association, Biogen, Eisai, Grifols, Harvard Medical School Post Graduate Education, NIH, Novo Nordisk, Roche/ Genentech, Suven, and Synexus; and royalties from Oxford University Press.

Ethical approval The study was approved by the Western Institutional Review Board. Research was completed in accordance with the Helsinki Declaration.

Informed consent All participants provided signed informed consent.

Consent for publication All authors agree to this publication.

Open Access This article is licensed under a Creative Commons Attribution 4.0 International License, which permits use, sharing, adaptation, distribution and reproduction in any medium or format, as long as you give appropriate credit to the original author(s) and the source, provide a link to the Creative Commons licence, and indicate if changes were made. The images or other third party material in this article are included in the article's Creative Commons licence, unless indicated otherwise in a credit line to the material. If material is not included in the article's Creative Commons licence and your intended use is not permitted by statutory regulation or exceeds the permitted use, you will 
need to obtain permission directly from the copyright holder. To view a copy of this licence, visit http://creativecommons.org/licenses/by/4.0/.

\section{References}

1. United Nations Department of Economic and Social Affairs Population Division. World Population Prospects: The 2017. Revision, custom data acquired via web-site. 2017.

2. Corrada MM, Brookmeyer R, Paganini-Hill A et al (2010) Dementia incidence continues to increase with age in the oldest old: the 90+ study. Ann Neurol 67:114-121. https://doi.org/10. 1002/ana.21915

3. Cecato JF, Martinelli JE, Izbicki R et al (2017) A subtest analysis of The Montreal Cognitive Assessment (MoCA): which subtests can best discriminate between healthy controls, mild cognitive impairment and Alzheimer's disease? Int Psychogeriatr 29:701. https://doi.org/10.1017/S104161021600212X

4. Borland E, Nägga K, Nilsson PM et al (2017) The montreal cognitive assessment: normative data from a large Swedish populationbased cohort. J Alzheimers Dis 59:893-901. https://doi.org/10. 3233/JAD-170203

5. Cesar KG, Yassuda MS et al (2019) MoCA test: normative and diagnostic accuracy data for seniors with heterogeneous educational levels in Brazil. Arq Neuropsiquiatr 77:775-781. https:// doi.org/10.1590/0004-282X20190130

6. Nasreddine ZS, Phillips NA, Bédirian V et al (2005) The Montreal Cognitive Assessment, MoCA: a brief screening tool for mild cognitive impairment. J Am Geriatr Soc 53:695-699. https://doi. org/10.1111/j.1532-5415.2005.53221.x

7. Nasreddine ZS. Montreal cognitive assessment. www.mocatest. org (2019). Accessed Feb 272020.

8. Wittich W, Phillips N, Nasreddine ZS et al (2010) Sensitivity and specificity of the Montreal Cognitive Assessment modified for individuals who are visually impaired. J Visual Impairm Blindness 104:360-368

9. Zietemann V, Kopczak A, Müller C et al (2017) Validation of the telephone interview of cognitive status and telephone Montreal Cognitive Assessment against detailed cognitive testing and clinical diagnosis of mild cognitive impairment after stroke. Stroke 48:2952-2957. https://doi.org/10.1161/STROKEAHA.117. 017519

10. Pendlebury ST, Welch SJ, Cuthbertson FC et al (2013) Telephone assessment of cognition after transient ischemic attack and stroke: modified telephone interview of cognitive status and telephone Montreal Cognitive Assessment versus face-to-face Montreal Cognitive Assessment and neuropsychological battery. Stroke 44:227-229. https://doi.org/10.1161/STROKEAHA.112.673384

11. Lai Y, Jiang C, Du X, Wang Z, Zhang J, Liu X, et al. Validation of T-MoCA in the screening of mild cognitive impairment in Chinese patients with atrial fibrillation. BMC Geriatrics. 2020; Under review. https://doi.org/10.21203/rs.3.rs-18762/v1.

12. Benge JF, Kiselica AM (2020) Rapid communication: preliminary validation of a telephone adapted Montreal Cognitive Assessment for the identification of mild cognitive impairment in Parkinson's disease. Clin Neuropsychol 35:133-147. https://doi.org/10.1080/ 13854046.2020.1801848

13. Folstein MF, Folstein SE, McHugh PR (1975) "Mini-mental state". A practical method for grading the cognitive state of patients for the clinician. J Psychiatr Res 12:189-198

14. Luis CA, Keegan AP, Mullan M (2009) Cross validation of the Montreal Cognitive Assessment in community dwelling older adults residing in the Southeastern US. Int J Geriatr Psychiatry 24:197-201. https://doi.org/10.1002/gps.2101
15. Freitas S, Simoes MR, Alves L et al (2013) Montreal cognitive assessment: validation study for mild cognitive impairment and Alzheimer disease. Alzheimer Dis Assoc Disord 27:37-43. https://doi.org/10.1097/WAD.0b013e3182420bfe

16. Roalf DR, Moberg PJ, Xie SX et al (2013) Comparative accuracies of two common screening instruments for classification of Alzheimer's disease, mild cognitive impairment, and healthy aging. Alzheimer's Dementia 9:529-537. https://doi.org/10.1016/j.jalz. 2012.10.001

17. Trzepacz PT, Hochstetler H, Wang $S$ et al (2015) Initiative AsDN. Relationship between the Montreal Cognitive Assessment and Mini-mental State Examination for assessment of mild cognitive impairment in older adults. BMC Geriatr 15:107. https://doi.org/10.1186/s12877-015-0103-3

18. Helmi L, Meagher D, O'Mahony E et al (2016) Agreement and conversion formula between Mini-Mental State Examination and Montreal Cognitive Assessment in an outpatient sample. World J Psychiatry 22:358-364

19. Saczynski JS, Inouye SK, Guess J et al (2015) The montreal cognitive assessment: creating a crosswalk with the mini-mental state examination. J Am Geriatr Soc 63:2370-2374. https://doi. org/10.1111/jgs. 13710

20. Katz MJ, Wang C, Nester CO et al (2021) T-MoCA: A valid phone screen for cognitive impairment in diverse community samples. Alzheimers Dement (Amst) 13(1):e12144. https://doi. org/10.1002/dad2.12144.

21. O'Connor K, Coon DW, Malek-Ahmadi M et al (2016) Description and cohort characterization of the Longevity Study: learning from our elders. Aging Clin Exp Res 28:863-869. https://doi.org/ 10.1007/s40520-015-0488-z

22. O'Bryant SE, Humphreys JD, Smith GE et al (2008) Detecting Dementia with the mini-mental state examination in highly educated individuals. Arch Neurol 65:963-967. https://doi.org/10. 1001/archneur.65.7.963

23. Spering CC, Hobson V, Lucas JA et al (2012) Diagnostic accuracy of the MMSE in detecting probable and possible Alzheimer's disease in ethnically diverse highly educated individuals: an analysis of the NACC database. J Gerontol A Biol Sci Med Sci 67:890-896. https://doi.org/10.1093/gerona/gls006

24. Radloff LS (1977) The CES-D scale: a self report depression scale for research in the general population. Appl Psychol Meas 1:385-401

25. Whittle C, Corrada MM, Dick M et al (2007) Neuropsychological data in nondemented oldest old: the 90+ Study. J Clin Exp Neuropsychol 29:290-299. https://doi.org/10.1080/1380339060 0678038

26. Melikyan ZA, Corrada MM, Dick MB et al (2019) Neuropsychological test norms in cognitively intact oldest-old. J Int Neuropsychol Soc 25:530-545. https://doi.org/10.1017/S13556177190001 22

27. van Steenoven I, Aarsland D, Hurtig H et al (2014) Conversion between mini-mental state examination, montreal cognitive assessment, and dementia rating scale- 2 scores in Parkinson's disease. Mov Disord 29:1809-1815. https://doi.org/10.1002/mds. 26062

28. Bergeron D, Flynn K, Verret L et al (2017) Multicenter validation of an MMSE-MoCA conversion table. J Am Geriatr Soc 65:1067-1072. https://doi.org/10.1111/jgs. 14779

29. Lawton M, Kasten M, May MT et al (2016) Validation of conversion between mini-mental state examination and montreal cognitive assessment. Mov Disord 31:593-596. https://doi.org/10.1002/ mds. 26498

30. Wong A, Black SE, Yiu SYP et al (2018) Converting MMSE to MoCA and MoCA 5-minute protocol in an educationally heterogeneous sample with stroke or transient ischemic attack. Int J Geriatr Psychiatry 33:729-734. https://doi.org/10.1002/gps.4846 
31. Kolen MJ, Brennan RL (2006) Test equating, scaling and linking: methods and practices, 2nd edn. Springer, New York

32. R Core Team (2017) R: a language and environment for statistical computing. R Foundation for Statistical Computing, Vienna

33. Albano AD (2016) Equate: an R Package for observed-score linking and equating. J Stat Softw 74:1-36

34. Dharmasaroja PA, Charernboon T, Assanasen J et al (2020) The correlation of Thai Mental State Examination (TMSE) and Montreal Cognitive Assessment (MoCA) and conversion table. J Med Assoc Thai 103:66-69

35. Larner AJ (2017) Converting cognitive screening instrument test scores to MMSE scores: regression equations. Int J Geriatr Psychiatry 32:351-352. https://doi.org/10.1002/gps.4622

36. Solomon TM, Debros GB, Budson AE et al (2014) Correlational analysis of 5 commonly used measures of cognitive functioning and mental status. Am J Alzheimer's Dis Other Demen 29:718722. https://doi.org/10.1177/1533317514534761

37. Yu RL, Lee WJ, Li JY et al (2020) Evaluating mild cognitive dysfunction in patients with Parkinson's disease in clinical practice in Taiwan. Sci Rep 10:1014. https://doi.org/10.1038/ s41598-020-58042-2

38. Nasreddine Z (2010) Montreal cognitive assessment (MoCA) BLIND. https://www.mocatest.org/

39. He W, Muenchrath M (2011) ACS-17 90+ in the United States: 2006-2008. American Community Survey Reports. Washington, D.C, US Census Bureau

40. Ardila A (2007) Toward the development of a cross-linguistic naming test. Arch Clin Neuropsychol 22:297-307

41. D'Elia LF, Satz P, Schretlen D (1989) Wechsler memory scale: a critical appraisal of the normative studies. J Clin Exp Neuropsychol 11:551-568. https://doi.org/10.1080/01688638908400913

Publisher's Note Springer Nature remains neutral with regard to jurisdictional claims in published maps and institutional affiliations. 